RAFAŁ KuCHARSKI (Warszawa)

\title{
CONVERGENCE OF OPTIMAL STRATEGIES IN A DISCRETE TIME MARKET WITH FINITE HORIZON
}

Abstract. A discrete-time financial market model with finite time horizon is considered, together with a sequence of investors whose preferences are described by a convergent sequence of strictly increasing and strictly concave utility functions. Existence of unique optimal consumption-investment strategies as well as their convergence to the limit strategy is shown.

Introduction. Recently, in a number of papers the following question was considered: does convergence of investors' preferences imply the convergence of their optimal strategies? In [2] a model with complete Brownian market model was described, while in [1] a discrete time model with finite horizon and utility functions defined on the whole real line was studied. Both papers gave a positive answer to the above problem under suitable assumptions.

In the present paper we prove a similar result for a discrete time market model with a finite horizon. We assume weaker regularity conditions on utility functions: strict concavity and strict monotonicity. The utility functions considered are defined on the positive axis.

In the first section we describe our model of financial market. Then we consider a one-step model and utilizing ideas from [4], we establish a few useful technical results. Finally, we prove the existence of optimal strategies for our model and their convergence together with the convergence of the investors' preferences.

1. Market model. Let $\left(\Omega, \mathcal{F},\left(\mathcal{F}_{t}\right)_{0 \leq t \leq T}, \mathbb{P}\right)$ be a discrete-time filtered probability space with finite time horizon $T \in \mathbb{N}$, with $\mathcal{F}_{0}=\{\emptyset, \Omega\}$. Prices of

2000 Mathematics Subject Classification: 49L20, 91B16, 91B28, 93E20.

Key words and phrases: utility maximization, dynamic programming, optimal strategies. 
$d$ risky securities available on the market are represented by a $d$-dimensional, almost surely positive adapted process $S_{t}=\left(S_{t, 1}, \ldots, S_{t, d}\right)^{\prime}, 0 \leq t \leq T$. For $t=0, \ldots, T-1$ we define

$$
\zeta_{t, i}=\frac{S_{t+1, i}}{S_{t, i}}, \quad i=1, \ldots, d,
$$

and $\zeta_{t}=\left(\zeta_{t, 1}, \ldots, \zeta_{t, d}\right)^{\prime}$. Let $D_{t}(\omega)$ be the smallest linear subspace containing the support of the regular conditional distribution of $\zeta_{t}$ with respect to $\mathcal{F}_{t}$ (it exists, cf. [6, Theorem 2.7.5]). Throughout the paper we assume that there are no redundant assets on the market, thus we have the following non-degeneracy assumption:

Assumption 1.1. $D_{t}$ is almost surely equal to $\mathbb{R}^{d}$ for $0 \leq t \leq T-1$.

Let $\Delta_{0}=\left\{\nu \in \mathbb{R}^{d}: \nu_{i} \geq 0, \sum_{i=1}^{d} \nu_{i} \leq 1\right\}$, and $\Delta=\left\{\nu \in \Delta_{0}: \sum_{i=1}^{d} \nu_{i}=1\right\}$. We denote by $\langle\cdot, \cdot\rangle$ the usual scalar product in $\mathbb{R}^{d}$. Denote by $X_{t}$ the wealth process at time $t$ before consumption and possible transactions. Let $\pi_{t, i}$ and $\bar{\pi}_{t, i}$ be the portions of the wealth $X_{t}$ invested in the $i$ th asset at time $t$ before and respectively after consumption and possible transactions. We do not allow short selling or short borrowing, so $\pi_{t}=\left(\pi_{t, 1}, \ldots, \pi_{t, d}\right)^{\prime} \in \Delta$ and $\bar{\pi}_{t}=\left(\bar{\pi}_{t, 1}, \ldots, \bar{\pi}_{t, d}\right)^{\prime} \in \Delta_{0}$.

At time $t=0, \ldots, T-1$, the investor who owns initial wealth $X_{t}$ invested in portfolio $\pi_{t}$, consumes a part $\alpha_{t} \in[0,1]$ of his wealth and changes his portfolio composition to $\bar{\pi}_{t}$, according to the equation

$$
X_{t}=X_{t} \alpha_{t}+X_{t} \sum_{i=1}^{d} \bar{\pi}_{t, i},
$$

which implies that we are interested only in $\mathcal{F}_{t}$-measurable strategies such that $\left(\alpha_{t}, \bar{\pi}_{t}\right) \in[0,1] \times \Delta_{0}$ a.s. and

$$
\alpha_{t}+\sum_{i=1}^{d} \bar{\pi}_{t, i}=1 \quad \text { a.s. }
$$

Denote the set of such strategies by $\mathcal{A}_{t}$.

At time $t+1$, due to price changes, the investor's wealth changes to

$$
X_{t+1}=\sum_{i=1}^{d} X_{t} \bar{\pi}_{t, i} \zeta_{t, i}=X_{t}\left\langle\bar{\pi}_{t}, \zeta_{t}\right\rangle .
$$

Equation (1.2) describes the dynamics of the control system we are dealing with: $X_{t}$ is regarded as a state of the system, and $\left(\alpha_{t}, \bar{\pi}_{t}\right) \in[0,1] \times \Delta_{0}$ are its control parameters, constrained by (1.1) describing the admissible strategies. The initial condition is given by the endowment $x:=X_{0}>0$. 
We consider a sequence of investors with preferences described by utility functions $U_{t}^{n}:(0, \infty) \rightarrow \mathbb{R}, 0 \leq t \leq T, n \in \overline{\mathbb{N}}:=\mathbb{N} \cup\{\infty\}$.

Assumption 1.2. The functions $U_{t}^{n}$ are strictly increasing and strictly concave for $t \in\{0, \ldots, T\}$ and $n \in \overline{\mathbb{N}}$. Moreover, for all $t \in\{0, \ldots, T\}$ and $x \in(0, \infty)$,

$$
U_{t}^{n}(x) \rightarrow U_{t}^{\infty}(x) \quad \text { as } n \rightarrow \infty .
$$

We are interested in maximization of the expected utility from consumption and terminal wealth, that is, we want to maximize the following reward functional:

$$
J_{T}^{n}(x,(\alpha, \bar{\pi}))=\mathbb{E}\left(\sum_{t=0}^{T-1} U_{t}^{n}\left(X_{t} \alpha_{t}\right)+U_{T}^{n}\left(X_{T}\right)\right) .
$$

For our dynamic programming problem to be well posed and finite, we assume that the following conditions are satisfied:

Assumption 1.3. For all $n \in \overline{\mathbb{N}}, k \in\{1, \ldots, T\}$ and $x>0$,

$$
\begin{aligned}
& \mathbb{E}\left(U_{k}^{n}\right)^{+}\left(x \prod_{t=0}^{k-1} \max \left\{\zeta_{t, i}: i=1, \ldots, d\right\}\right)<\infty, \\
& \mathbb{E}\left(U_{k}^{n}\right)^{-}\left(x \prod_{t=0}^{k-1} \min \left\{\zeta_{t, i}: i=1, \ldots, d\right\}\right)<\infty .
\end{aligned}
$$

REMARK 1.4. One can consume all or nothing of the wealth, so we need values of utility functions at 0 . We deal with that problem by putting $U(0):=$ $\lim _{x \rightarrow 0^{+}} U(x)$; if this limit is finite, the continuity and concavity properties are kept, and if not (e.g. for a logarithmic function), the agent will not choose such a strategy to maximize utility.

2. One-step case. We start with the case $T=1$. Let $u, v:(0, \infty) \rightarrow \mathbb{R}$ be strictly increasing functions, $u$ strictly concave and $v$ concave. Let $\mathcal{H}$ be a sub- $\sigma$-field of $\mathcal{F}$, let $\zeta=\left(\zeta_{1}, \ldots, \zeta_{d}\right)^{\prime}$ be an $\mathbb{R}^{d}$-valued random variable with non-degenerate (in the sense of Assumption 1.1) conditional distribution with respect to $\mathcal{H}$, and let $\mathbb{E}(\cdot \mid \mathcal{H})$ denote conditional expectation with respect to $\mathcal{H}$. Denote by $\mathcal{A}$ the set of admissible strategies: $\mathcal{H}$-measurable random variables such that $(\alpha, \bar{\pi}) \in[0,1] \times \Delta_{0}$ a.s. and $\alpha+\sum_{j=1}^{d} \bar{\pi}_{j}=1$. Define the value function by

$$
w(x):=\operatorname{ess}_{(\alpha, \bar{\pi}) \in \mathcal{A}}\{u(x \alpha)+\mathbb{E}(v(x\langle\bar{\pi}, \zeta\rangle) \mid \mathcal{H})\}, \quad x>0 .
$$

Analogously to Assumption 1.3, we introduce 
Assumption 2.1. For all $x>0$,

$$
\mathbb{E} v^{+}\left(x \max _{i \in\{1, \ldots, d\}} \zeta_{i}\right)<\infty \quad \text { and } \quad \mathbb{E} v^{-}\left(x \min _{i \in\{1, \ldots, d\}} \zeta_{i}\right)<\infty .
$$

The following technical lemmas are crucial:

LEMMA 2.2. There exists an almost surely continuous, strictly concave and strictly increasing (with respect to every coordinate) version of

$$
[0, \infty)^{d} \backslash\{0\} \ni \pi \mapsto \mathbb{E}(v(\langle\pi, \zeta\rangle) \mid \mathcal{H}) .
$$

Proof. Let $\kappa$ denote the regular conditional distribution of $\zeta$ given $\mathcal{H}$. Then

$$
\mathbb{E}(v(\langle\pi, \zeta\rangle) \mid \mathcal{H})=\int_{\mathbb{R}^{d}} v(\langle\pi, x\rangle) \kappa(d x) \quad \text { a.s. },
$$

and we take the right side as a definition of our version. By a routine calculation one checks it has the desired properties. We will show concavity. Fix $\pi^{1}, \pi^{2} \in[0, \infty)^{d} \backslash\{0\}, \pi^{1} \neq \pi^{2}$ and $t \in(0,1)$. Then

$$
\begin{aligned}
t \mathbb{E}\left(v\left(\left\langle\pi^{1}, \zeta\right\rangle\right) \mid \mathcal{H}\right)+(1-t) \mathbb{E}(v & \left.\left(\left\langle\pi^{2}, \zeta\right\rangle\right) \mid \mathcal{H}\right) \\
& =\int_{\mathbb{R}^{d}}\left[t v\left(\left\langle\pi^{1}, x\right\rangle\right)+(1-t) v\left(\left\langle\pi^{2}, x\right\rangle\right)\right] \kappa(d x) \\
& <\int_{\mathbb{R}^{d}} v\left(\left\langle t \pi^{1}+(1-t) \pi^{2}, x\right\rangle\right) \kappa(d x) \\
& =\mathbb{E}\left(v\left(\left\langle t \pi^{1}+(1-t) \pi^{2}, \zeta\right\rangle\right) \mid \mathcal{H}\right) \quad \text { a.s. }
\end{aligned}
$$

The strict inequality is justified by Assumption 1.1.

Proposition 2.3. For every $x \in(0, \infty)$ there exists a unique optimal pair $(\widehat{\alpha}, \widehat{\pi}) \in \mathcal{A}$ such that

$$
w(x)=u(x \widehat{\alpha})+\mathbb{E}(v(x\langle\widehat{\pi}, \zeta\rangle) \mid \mathcal{H}) \quad \text { a.s. }
$$

Proof. We take the version of conditional expectation with the properties stated in Lemma 2.2, and consider the mapping

$$
\Phi:[0,1] \times \Delta_{0} \times \Omega \ni(\alpha, \bar{\pi}, \omega) \mapsto u(x \alpha)+\mathbb{E}(v(x\langle\bar{\pi}, \zeta\rangle) \mid \mathcal{H})(\omega) \in \mathbb{R}
$$

which is continuous except on a $\mathbb{P}$-zero set $N$. Since the set

$$
\left\{(\alpha, \bar{\pi}) \in[0,1] \times \Delta_{0}: \alpha+\sum_{j=1}^{d} \bar{\pi}_{j}=1\right\}
$$

is compact, for any $\omega \in \Omega \backslash N$ there is a pair $(\widehat{\alpha}(\omega), \widehat{\pi}(\omega))$ attaining the supremum of $\Phi$.

Suppose that there are two such pairs, say $\left(\alpha^{1}, \bar{\pi}^{1}\right),\left(\alpha^{2}, \bar{\pi}^{2}\right) \in \mathcal{A}$. Take any $t \in(0,1)$. Putting $\alpha=t \alpha^{1}+(1-t) \alpha^{2}, \bar{\pi}=t \bar{\pi}^{1}+(1-t) \bar{\pi}^{2}$ we have 
$\alpha \in(0,1), \bar{\pi} \in \Delta_{0}$ a.s. Since $\sum_{i=1}^{m} \bar{\pi}_{i}=1-\alpha$, it follows that $(\alpha, \bar{\pi}) \in \mathcal{A}$ and

$$
\begin{aligned}
w(x)= & t w(x)+(1-t) w(x) \\
= & t\left[u\left(x \alpha^{1}\right)+\mathbb{E}\left(v\left(x\left\langle\bar{\pi}^{1}, \zeta\right\rangle\right) \mid \mathcal{H}\right)\right] \\
& +(1-t)\left[u\left(x \alpha^{2}\right)+\mathbb{E}\left(v\left(x\left\langle\bar{\pi}^{2}, \zeta\right\rangle\right) \mid \mathcal{H}\right)\right] \\
\leq & u(x \alpha)+\mathbb{E}\left(v\left(t x\left\langle\bar{\pi}^{1}, \zeta\right\rangle+(1-t) x\left\langle\bar{\pi}^{2}, \zeta\right\rangle\right) \mid \mathcal{H}\right) \\
= & u(x \alpha)+\mathbb{E}(v(x\langle\bar{\pi}, \zeta\rangle) \mid \mathcal{H}) \leq w(x) \quad \text { a.s. }
\end{aligned}
$$

Both $u$ and $v$ are strictly concave, thus the above inequality turns into an equality iff $\alpha^{1}=\alpha^{2}$ and $\left\langle\bar{\pi}^{1}, \zeta\right\rangle=\left\langle\bar{\pi}^{2}, \zeta\right\rangle$ a.s. From the assumption we made on the support of the distribution of $\zeta$, that implies $\bar{\pi}_{i}^{1}=\bar{\pi}_{i}^{2}$ a.s., $i=1, \ldots, d$, hence the proof of uniqueness is finished.

The optimal pair $(\widehat{\alpha}, \widehat{\pi})$ is an $\mathcal{H}$-measurable random variable, since for any open ball $B \subset \mathbb{R}^{d+1}$,

$$
(\widehat{\alpha}, \widehat{\pi})(\omega) \in B \Leftrightarrow \bigvee_{\left(\alpha^{*}, \pi^{*}\right) \in C \cap B} \bigwedge_{(\alpha, \pi) \in C \backslash B} \Phi\left(\alpha^{*}, \pi^{*}\right)(\omega)>\Phi(\alpha, \pi)(\omega)
$$

where $C$ denotes a countable dense subset of (2.2), and therefore

$$
\{(\widehat{\alpha}, \widehat{\pi}) \in B\}=\bigcup_{\left(\alpha^{*}, \pi^{*}\right) \in C \cap B} \bigcap_{(\alpha, \pi) \in C \backslash B}\left\{\Phi\left(\alpha^{*}, \pi^{*}\right)>\Phi(\alpha, \pi)\right\} \in \mathcal{H} .
$$

LEMMA 2.4. There is a version of the value function $w$ which is almost surely strictly increasing and strictly concave.

Proof. For every $q \in(0, \infty) \cap \mathbb{Q}$ fix a version of $w(q)$, which by Assumption 2.1 is almost surely finite. Fix $x, y \in(0, \infty) \cap \mathbb{Q}$. It is obvious that if $y<x$ then $w(y)<w(x)$ a.s. To show strict concavity, fix $t \in(0,1) \cap \mathbb{Q}$ and let $\left(\alpha^{x}, \bar{\pi}^{x}\right),\left(\alpha^{y}, \bar{\pi}^{y}\right) \in \mathcal{A}$ be optimal pairs for $x$ and $y$ respectively. Put $z=t x+(1-t) y, \beta=t x / z, \alpha=\beta \alpha^{x}+(1-\beta) \alpha^{y}, \bar{\pi}=\beta \bar{\pi}^{x}+(1-\beta) \bar{\pi}^{y}$. Obviously $\alpha \in[0,1], \beta \in(0,1)$ a.s. Since $\sum_{i=1}^{d} \bar{\pi}_{i}=1-\alpha$, we obtain

$$
t x \bar{\pi}^{x}+(1-t) y \bar{\pi}^{y}=z\left(\beta \bar{\pi}^{x}+(1-\beta) \bar{\pi}^{y}\right)=z \bar{\pi},
$$

and since $u$ and $v$ are strictly concave and $\zeta$ is almost surely positive, we have

$$
\begin{aligned}
t w(x)+(1-t) w(y)= & t\left[u\left(x \alpha^{x}\right)+\mathbb{E}\left(v\left(x\left\langle\bar{\pi}^{x}, \zeta\right\rangle\right) \mid \mathcal{H}\right)\right] \\
& +(1-t)\left[u\left(y \alpha^{y}\right)+\mathbb{E}\left(v\left(y\left\langle\bar{\pi}^{y}, \zeta\right\rangle\right) \mid \mathcal{H}\right)\right] \\
\leq & u(z \alpha)+\mathbb{E}(v(z\langle\bar{\pi}, \zeta\rangle \mid \mathcal{H})) \leq w(z) \quad \text { a.s. }
\end{aligned}
$$

and moreover this inequality turns into an equality iff

$$
x \alpha^{x}=y \alpha^{y} \quad \text { and } \quad x\left\langle\bar{\pi}^{x}, \zeta\right\rangle=y\left\langle\bar{\pi}^{y}, \zeta\right\rangle \quad \text { a.s. }
$$

Once again using our assumption on the distribution of $\zeta$, this implies

$$
x \bar{\pi}_{i}^{x}=y \bar{\pi}_{i}^{y}, \quad i=1, \ldots, d,
$$


and summing those equalities up for $i=1, \ldots, d$ we obtain

$$
x\left[1-\alpha^{x}\right]=y\left[1-\alpha^{y}\right],
$$

hence also $x=y$. This shows in particular that for all $x, y \in(0, \infty) \cap \mathbb{Q}$, $x \neq y$, we have

$$
w\left(\frac{x+y}{2}\right)>\frac{w(x)+w(y)}{2} \quad \text { a.s. }
$$

We can now extend this version of $w$ to a function which is almost surely strictly increasing and strictly continuous for all $x \in(0, \infty)$. Finally, from monotone convergence, for fixed $x \in(0, \infty)$ and a sequence of rationals $q_{n} \uparrow x$ we have

$$
\begin{aligned}
w(x)=\lim _{n} w\left(q_{n}\right) & =\lim _{n} \operatorname{esssup}_{(\pi, \alpha) \in \mathcal{A}}\left\{u\left(q_{n} \alpha\right)+\mathbb{E}\left(v\left(q_{n}\langle\bar{\pi}, \zeta\rangle\right) \mid \mathcal{H}\right)\right\} \\
& =\operatorname{ess}_{(\pi, \alpha) \in \mathcal{A}}\{u(x \alpha)+\mathbb{E}(v(x\langle\bar{\pi}, \zeta\rangle) \mid \mathcal{H})\} .
\end{aligned}
$$

Proposition 2.5. There exists a selector of optimal strategies

$$
(0, \infty) \in x \mapsto(\widehat{\alpha}, \widehat{\pi})(x) \in \mathcal{A}
$$

which is continuous for almost all $\omega$.

Proof. We fix a version of conditional expectation with the properties stated in Lemma 2.2. The random function

$$
w(x,(\alpha, \bar{\pi})):=u(x \alpha)+\mathbb{E}(v(x\langle\bar{\pi}, \zeta\rangle) \mid \mathcal{H})
$$

is then almost surely continuous, jointly for all arguments. Suppose there exists $x \in(0, \infty)$ and a sequence $x_{n} \in(0, \infty), n \in \mathbb{N}$, such that $x_{n} \rightarrow x$ and $(\widehat{\alpha}, \widehat{\pi})\left(x_{n}\right) \nrightarrow(\widehat{\alpha}, \widehat{\pi})(x)$. Since all $(\widehat{\alpha}, \widehat{\pi})\left(x_{n}\right)$ belong to the compact set $(2.2)$, we may choose, using Lemma 2 from [3], a random subsequence $(\widehat{\alpha}, \widehat{\pi})\left(x_{n_{k}}\right)$ converging to some $(\widetilde{\alpha}, \widetilde{\pi})$. Condition (1.1) holds for all $k \in \mathbb{N}$, so letting $k \rightarrow \infty$, we get $(\widetilde{\alpha}, \widetilde{\pi}) \in \mathcal{A}$. By continuity,

$$
\begin{aligned}
& \lim _{k \rightarrow \infty} w\left(x_{n_{k}},(\widehat{\alpha}, \widehat{\pi})\left(x_{n_{k}}\right)\right)=w(x,(\widetilde{\alpha}, \widetilde{\pi}))=: \widetilde{w}, \\
& \lim _{n \rightarrow \infty} w\left(x_{n},(\widehat{\alpha}, \widehat{\pi})(x)\right)=w(x,(\widehat{\alpha}, \widehat{\pi})(x))=: w,
\end{aligned}
$$

and if $(\widetilde{\alpha}, \widetilde{\pi}) \neq(\widehat{\alpha}, \widehat{\pi})(x)$, then $\widetilde{w}<w$. If we fix $\varepsilon \in(0,(w-\widetilde{w}) / 2)$, then for $k$ large enough

$$
w\left(x_{n_{k}},(\widehat{\alpha}, \widehat{\pi})(x)\right)>w-\varepsilon>\widetilde{w}+\varepsilon,
$$

while from (2.3),

$$
w\left(x_{n_{k}},(\widehat{\alpha}, \widehat{\pi})\left(x_{n_{k}}\right)\right)<\widetilde{w}+\varepsilon .
$$

Inequalities (2.5) and (2.6) lead to

$$
w\left(x_{n_{k}},(\widehat{\alpha}, \widehat{\pi})(x)\right)>w\left(x_{n_{k}},(\widehat{\alpha}, \widehat{\pi})\left(x_{n_{k}}\right)\right)
$$

contradicting the optimality of $(\widehat{\alpha}, \widehat{\pi})\left(x_{n_{k}}\right)$. 
3. Convergence of optimal strategies. We are now going to use the results of the previous section in the general case. We define the Bellman functions:

$$
\begin{aligned}
V_{T}^{n}(x) & :=U_{T}(x), \\
V_{t}^{n}(x):= & \operatorname{ess}_{(\alpha, \bar{\pi}) \in \mathcal{A}}\left\{U_{t}^{n}(\alpha x)+\mathbb{E}\left(V_{t+1}^{n}\left(x\left\langle\bar{\pi}, \zeta_{t}\right\rangle\right) \mid \mathcal{F}_{t}\right)\right\}
\end{aligned}
$$

for $x \in(0, \infty)$ and $t=0, \ldots, T-1$.

TheOREM 3.1. For all $n \in \overline{\mathbb{N}}$ and $t=0, \ldots, T$ :

(i) the function $V_{t}^{n}$ has a version which is strictly increasing and strictly concave almost surely,

(ii) there exists a unique $\mathcal{B}(0, \infty) \otimes \mathcal{F}_{t}$-measurable function $\left(\widehat{\alpha}_{t}^{n}, \widehat{\pi}_{t}^{n}\right) \in \mathcal{A}_{t}$ such that for all $x \in(0, \infty)$,

$$
V_{t}^{n}(x)=U_{t}^{n}\left(x \widehat{\alpha}_{t}^{n}(x)\right)+\mathbb{E}\left(V_{t+1}^{n}\left(x\left\langle\widehat{\pi}_{t}^{n}(x), \zeta_{t}\right\rangle\right) \mid \mathcal{F}_{t}\right) .
$$

Proof. Fix $n \in \overline{\mathbb{N}}$ and use backward induction. It is clear that $V_{T}^{n}$ is strictly concave and strictly increasing since $U_{T}^{n}$ is. Then decreasing $t$ from $T-1$ to 0 and applying Lemma 2.4 and Proposition 2.3 with $w:=V_{t}^{n}$, $u:=U_{t}^{n}, v:=V_{t+1}^{n}, \mathcal{A}:=\mathcal{A}_{t}, \mathcal{H}:=\mathcal{F}_{t}$ and $\zeta:=\zeta_{t}$, we find that $V_{t}^{n}$ has a strictly increasing and strictly concave version, and there is a unique optimal strategy $\left(\widehat{\alpha}_{t}^{n}, \widehat{\pi}_{t}^{n}\right):=(\widehat{\alpha}, \widehat{\pi})$ which is $\mathcal{F}_{t}$-measurable for all $x \in(0, \infty)$ and almost surely continuous, hence $\mathcal{B}(0, \infty) \otimes \mathcal{F}_{t}$-measurable. This proves the theorem.

In this section we will make repeated use of the following elementary fact. It may be derived e.g. from pages 90 and 248 of [5], but we include an easy proof for completeness.

LEMMA 3.2. Let $U \subset \mathbb{R}$ be an open set and $f_{n}: U \rightarrow \mathbb{R}$ be a sequence of increasing functions such that $f_{n}$ converges pointwise on $U$ to a continuous function $f$. Then $f_{n}$ converges to $f$ uniformly on each compact subset of $U$.

Proof. First notice that $f$ is increasing, being the limit of a sequence of increasing functions. Fix a compact set $C \subset U$ and an arbitrary $\varepsilon>0$. Without loss of generality, we may assume that $C=[a, b]$ is an interval. On $C$, the function $f$ is uniformly continuous, hence we can find $x_{0}, \ldots, x_{k} \in C$ with $a:=x_{0}<x_{1}<\cdots<x_{k-1}<x_{k}=: b$ such that $\left|f\left(x_{i}\right)-f\left(x_{i-1}\right)\right|<\varepsilon / 2$ for $i \in\{1, \ldots, k\}$. Let $N_{i} \in \mathbb{N}$ be such that $\left|f_{n}\left(x_{i}\right)-f\left(x_{i}\right)\right|<\varepsilon / 2$ for $n \geq N_{i}$, and define $N:=\max \left\{N_{i}: i \in\{0, \ldots, k\}\right\}$. Then for any $x \in A$ there is $i \in\{0, \ldots, k-1\}$ such that $x \in\left[x_{i}, x_{i+1}\right]$, and for $n \geq N$ we have

$$
\begin{aligned}
f(x)-\varepsilon \leq f\left(x_{i+1}\right)-\varepsilon & \leq f_{n}\left(x_{i+1}\right)-\varepsilon / 2 \leq f_{n}(x) \leq f_{n}\left(x_{i}\right)+\varepsilon / 2 \\
& \leq f\left(x_{i}\right)+\varepsilon \leq f(x)+\varepsilon
\end{aligned}
$$

Since $x \in C$ was arbitrary, the assertion follows. 
Now we are ready to prove the convergence of optimal strategies. Again we will start with the one-step case.

Proposition 3.3. Assume that for every $n \in \overline{\mathbb{N}}$ functions $u^{n}$, $v^{n}$ are strictly increasing and strictly concave, and moreover $\lim _{n \rightarrow \infty} u^{n}(x)=u^{\infty}(x)$ and $\lim _{n \rightarrow \infty} v^{n}(x)=v^{\infty}(x)$ for all $x \in(0, \infty)$. Let $\left(\widehat{\alpha}^{n}, \widehat{\pi}^{n}\right)$ denote the optimal strategy fulfilling (2.1) with $u$ and $v$ replaced by $u^{n}$ and $v^{n}$. Then, for every $x \in(0, \infty)$,

$$
\lim _{n \rightarrow \infty}\left(\widehat{\alpha}^{n}, \widehat{\pi}^{n}\right)(x)=\left(\widehat{\alpha}^{\infty}, \widehat{\pi}^{\infty}\right)(x) \quad \text { a.s. }
$$

Proof. Suppose that, on the contrary, the convergence fails for some $x \in$ $(0, \infty)$. Since $[0,1] \times \Delta_{0}$ is compact, by the use of Lemma 2 from [3] we choose a random subsequence $\left(n_{k} \in \mathbb{N}: k \in \mathbb{N}\right)$ such that $\lim _{k \rightarrow \infty}\left(\widehat{\alpha}^{n_{k}}, \widehat{\pi}^{n_{k}}\right)(x)=$ $(\widetilde{\alpha}, \widetilde{\pi}) \in \mathcal{A},(\widetilde{\alpha}, \widetilde{\pi}) \neq\left(\widehat{\alpha}^{\infty}, \widehat{\pi}^{\infty}\right)$. Define

$$
w^{n}(\alpha, \bar{\pi}):=u^{n}(x \alpha)+\mathbb{E} v^{n}(x\langle\bar{\pi}, \zeta\rangle \mid \mathcal{H}), \quad(\alpha, \bar{\pi}) \in \mathcal{A}, n \in \overline{\mathbb{N}},
$$

with a continuous version of the conditional expectation. Then the functions $w^{n}$ depend continuously on $\bar{\pi}$ and $\alpha$, the uniform convergence of $u^{n}$ and $v^{n}$ on compact sets gives

$$
\lim _{k \rightarrow \infty} w^{n_{k}}\left(\widehat{\alpha}^{n_{k}}, \widehat{\pi}^{n_{k}}\right)=w^{\infty}(\widetilde{\alpha}, \widetilde{\pi}) \quad \text { a.s. }
$$

and by our hypothesis

$$
\widetilde{w}:=w^{\infty}(\widetilde{\alpha}, \widetilde{\pi})<w^{\infty}\left(\widehat{\alpha}^{\infty}, \widehat{\pi}^{\infty}\right)=: w .
$$

Fix $\varepsilon \in(0,(w-\widetilde{w}) / 2)$. Since pointwise convergence ensures

$$
\lim _{n \rightarrow \infty} w^{n}\left(\widehat{\alpha}^{\infty}, \widehat{\pi}^{\infty}\right)=w
$$

for $k$ large enough we have

$$
w^{k}\left(\widehat{\alpha}^{\infty}, \widehat{\pi}^{\infty}\right)>w-\varepsilon>\widetilde{w}+\varepsilon,
$$

while from (3.2) we get

$$
w^{n_{k}}\left(\widehat{\alpha}^{n_{k}}, \widehat{\pi}^{n_{k}}\right)<\widetilde{w}+\varepsilon
$$

Combining (3.3) and (3.4) we obtain $w^{n_{k}}\left(\widehat{\alpha}^{\infty}, \widehat{\pi}^{\infty}\right)>w^{n_{k}}\left(\widehat{\alpha}^{n_{k}}, \widehat{\pi}^{n_{k}}\right)$, contradicting the optimality of $\left(\widehat{\alpha}^{n_{k}}, \widehat{\pi}^{n_{k}}\right)$.

Now we can prove the main theorem.

THEOREM 3.4. Let $\left(\left(\widehat{\alpha}_{t}^{n}, \widehat{\pi}_{t}^{n}\right): t=0, \ldots, T-1\right)$ be optimal strategies maximizing (1.3) with the corresponding functions $\left(U_{0}^{n}, \ldots, U_{T}^{n}\right), n \in \overline{\mathbb{N}}$. Then for every $x \in(0, \infty)$,

$$
\lim _{n \rightarrow \infty}\left(\widehat{\alpha}_{t}^{n}, \widehat{\pi}_{t}^{n}\right)(x)=\left(\widehat{\alpha}_{t}^{\infty}, \widehat{\pi}_{t}^{\infty}\right)(x) \quad \text { a.s., } t=0, \ldots, T-1 .
$$

Proof. The assertion follows from the foregoing proposition applied consecutively to the Bellman functions (3.1) with $u^{n}:=U_{t}^{n}$ and $v^{n}:=V_{t+1}^{n}$ for $t=T-1, \ldots, 0$. We only need to check that $\lim _{n \rightarrow \infty} V_{t}^{n}(x)=V_{t}^{\infty}(x)$ 
for $x \in(0, \infty)$ and $t=T, \ldots, 1$. For $t=T$ this is obvious since $V_{T}^{n}=U_{T}^{n}$, $n \in \overline{\mathbb{N}}$. If we have proved that $\left(\widehat{\alpha}_{t}^{n}, \widehat{\pi}_{t}^{n}\right) \rightarrow\left(\widehat{\alpha}_{t}^{\infty}, \widehat{\pi}_{t}^{\infty}\right)$ for some $t \leq T$, then from uniform convergence on compact sets and the Lebesgue Theorem, for all $x \in(0, \infty)$,

$$
\begin{aligned}
\lim _{n \rightarrow \infty} V_{t}^{n}(x) & =\lim _{n \rightarrow \infty}\left(U_{t}^{n}\left(x \widehat{\alpha}_{t}^{n}(x)\right)+\mathbb{E}\left(V_{t+1}^{n}\left(x\left\langle\widehat{\pi}_{t}^{n}(x), \zeta_{t}\right\rangle\right) \mid \mathcal{F}_{t}\right)\right) \\
& =U_{t}^{\infty}\left(x \widehat{\alpha}_{t}^{\infty}(x)\right)+\mathbb{E}\left(V_{t+1}^{\infty}\left(x\left\langle\widehat{\pi}_{t}^{\infty}(x), \zeta_{t}\right\rangle\right) \mid \mathcal{F}_{t}\right) \\
& =V_{t}^{\infty}(x) .
\end{aligned}
$$

\section{References}

[1] L. Carassus and M. Ràsonyi, Optimal strategies and utility-based prices converge when agents' preferences do, preprint.

[2] E. Jouini and C. Napp, Convergence of utility functions and convergence of optimal strategies, Finance Stoch. 8 (2004), 133-144.

[3] Yu. M. Kabanov and Ch. Stricker, A teachers' note on no-arbitrage criteria, in: Séminaire de Probabilités, XXXV, Lecture Notes in Math. 1755, Springer, Berlin, 2001, 149-152.

[4] M. Ràsonyi and . Stettner, On utility maximization in discrete-time financial market models, Ann. Appl. Probab. 15 (2005), 1367-1395.

[5] R. T. Rockafellar, Convex Analysis, Princeton Math. Ser. 28, Princeton Univ. Press, Princeton, NJ, 1970.

[6] A. N. Shiryaev, Probability, Grad. Texts in Math. 95, Springer, New York, 1996.

Institute of Mathematics

Polish Academy of Sciences

Śniadeckich 8

00-956 Warszawa, Poland

E-mail: R.Kucharski@impan.gov.pl

Received on 24.1.2006;

revised version on 10.3.2006 